TITLE:

\title{
Numerical verification of the random-phase-and-amplitude formalism of weak turbulence
}

$\operatorname{AUTHOR}(\mathrm{S})$ :

Tanaka, Mitsuhiro; Yokoyama, Naoto

\section{CITATION:}

Tanaka, Mitsuhiro ...[et al]. Numerical verification of the random-phase-and-amplitude formalism of weak turbulence. Physical Review E 2013, 87(6): 062922.

ISSUE DATE:

2013-06-27

URL:

http://hdl.handle.net/2433/188010

RIGHT:

(C)2013 American Physical Society 
PHYSICAL REVIEW E 87, 062922 (2013)

\title{
Numerical verification of the random-phase-and-amplitude formalism of weak turbulence
}

\author{
Mitsuhiro Tanaka* \\ Faculty of Engineering, Gifu University, Gifu 501-1193, Japan \\ Naoto Yokoyama \\ Department of Aeronautics and Astronautics, Kyoto University, Kyoto 615-8540, Japan
}

(Received 15 January 2013; published 27 June 2013)

\begin{abstract}
The random-phase-and-amplitude-formalism (RPA) has significantly extended the scope of weak turbulence studies. Because the RPA does not assume any proximity to the Gaussianity in the wave number space, it can predict, for example, how the fluctuation of the complex amplitude of each wave mode grows through nonlinear interactions with other modes and how it approaches the Gaussianity. Thus, the RPA has a great potential capability, but its validity has been assessed neither numerically nor experimentally. We compare the theoretical predictions given by the RPA with the results of direct numerical simulations (DNS) for a three-wave Hamiltonian system, thereby assessing the validity of the RPA. The predictions of the RPA agree quite well with the results of DNS in all the aspects of the statistical characteristics of the mode amplitudes studied here.
\end{abstract}

DOI: 10.1103/PhysRevE.87.062922

PACS number(s): 05.45.-a, 42.65.Wi, 47.27.-i

\section{INTRODUCTION}

In weak turbulence, wave trains which have different directions of the propagation, wavelengths, and frequencies weakly interact with each other owing to the nonlinearity in the governing equation and/or the boundary conditions. Many papers on weak turbulence have been devoted to the derivation of the kinetic equation [1-3], which governs the statistical evolution of the wave action spectrum, the physical and mathematical properties of the kinetic equation and resulting spectra [4-8], the statistically steady nonequilibrium spectra when the external forces and the dissipation balances $[9,10]$, and so on. Thus, research on weak turbulence has been conventionally focused on the wave action spectrum, which is the ensemble average of the squared norm of the complex amplitude of each mode [11]. On the other hand, the spectra which are observed in the direct numerical simulations (DNS) and field observations have large fluctuations. In DNS, the amplitude of each wave mode is often determined by a prescribed spectrum and initially has no fluctuations at all. Even in such DNS, the amplitude fluctuations spontaneously grow as time elapses. The generation mechanism of the fluctuations, the time scales of their growths, and the possibility of the approach to the Gaussianity had little been studied.

The random-phase-and-amplitude formalism (RPA) which has recently been developed has changed the situation and drastically extended the scope of weak turbulence research [12-15]. Similar to the conventional weak turbulence theory, the RPA is the statistical theory for the complex amplitude $a_{k}$. Let $a_{k}$ be expressed as $a_{k}=\left|a_{k}\right| \psi_{k}$ with the positive amplitude $\left|a_{k}\right|$ and the phase factor $\psi_{k}=e^{i \phi_{k}}$. In the random phase approximation (RP) in the strict sense, it is assumed that $\psi_{\boldsymbol{k}}$ for all $\boldsymbol{k}$ are independent random variables and are uniformly distributed over the unit circle in the complex plane. In the RPA it is assumed, in addition to the assumptions of the $\mathrm{RP}$, that the amplitudes $\left|a_{k}\right|$ also are mutually independent

\footnotetext{
*tanaka@gifu-u.ac.jp

†yokoyama@kuaero.kyoto-u.ac.jp
}

random variables for all $\boldsymbol{k}$. It is important to note that in the RPA $\left|a_{k}\right|$ are allowed to have any distribution and can be far from the Gaussianity. Although it is only recently that the RPA has been defined unambiguously, it has implicitly been used for a rather long time under the name of "the random phase approximation," without even realizing the important assumption about the amplitude statistics. For example, Zakharov et al. ([11], p. 65) calls their approximation "the random phase approximation," but they implicitly assume the statistical independence of the amplitude $\left|a_{k}\right|$ for different $\boldsymbol{k}$.

The equations which describe the time evolution of the moments of $\left|a_{k}\right|^{2}$ for arbitrary orders as well as the probability density function (PDF) of $\left|a_{k}\right|^{2}$ have been derived. Thus, the RPA is expected to describe the statistical characteristics of the amplitude fluctuations, which have little been studied. Although the RPA seems to have a great potential capability, its validity has been assessed neither numerically nor experimentally. In this study, we perform a series of large-scale DNS for a model Hamiltonian system, which allows threewave resonance, and compare the results with the theoretical predictions of the RPA. Here we confine our attention to the single-mode statistics of the amplitude fluctuations. In every aspect studied here, we have obtained good quantitative agreement between the RPA and the DNS.

Benney and Newell [16] investigated the $n$th order cumulant $R^{(n)}$ of a wave turbulence field in the physical space and derived an equation which governs the temporal evolution of the Fourier transform of $R^{(n)}$. They assumed that the wave field is weak nonlinear and that the medium is dispersive, but did not assume that the wave field is close to a Gaussian state in the physical space. Prior to Ref. [16], Benney and Saffman [17] derived the kinetic equation for the action spectrum based on the same assumptions. In the present paper, we compare our numerical results with the predictions of the RPA only and do not make any comparison with the predictions of Ref. [16] mainly for the following two reasons. First, [16] is described in terms of the Fourier transform of a real-valued physical variable, and it is not expressed in terms of the complex amplitude of wave modes which we want to handle. Second, Ref. [16] does not introduce the discretization of the $\boldsymbol{k}$ space 
and the Fourier transform is a generalized function. On the other hand, the RPA treats the discretized $\boldsymbol{k}$ space from the outset, hence the comparison with the DNS is straightforward and much easier than the case of Ref. [16]. There is also an essential difference between the RPA and the analysis in Refs. [16] and [17] with regard to the speed of decay of the correlations in the physical space as functions of the separation of points. (See, for example, Ref. [12].)

This paper is organized as follows. The numerical scheme is presented in Sec. II. In Sec. III, numerical results of the evolution of the amplitude fluctuations and the approach to the Gaussianity are reported. The discussion and the summary are given in Sec. IV.

\section{NUMERICAL SCHEME}

\section{A. Numerical model}

In this study we employ the following three-wave Hamiltonian system:

$$
\begin{aligned}
\mathcal{H}= & \mathcal{H}_{2}+\mathcal{H}_{3}, \\
\mathcal{H}_{2}= & \int \omega(\boldsymbol{k})|a(\boldsymbol{k})|^{2} d \boldsymbol{k}, \\
\mathcal{H}_{3}= & \frac{1}{2} \int\left[V\left(\boldsymbol{k}, \boldsymbol{k}_{1}, \boldsymbol{k}_{2}\right) a^{*}(\boldsymbol{k}) a\left(\boldsymbol{k}_{1}\right) a\left(\boldsymbol{k}_{2}\right)+\text { c.c. }\right] \\
& \times \delta\left(\boldsymbol{k}-\boldsymbol{k}_{1}-\boldsymbol{k}_{2}\right) d \boldsymbol{k}_{123} ; \\
\frac{d a(\boldsymbol{k})}{d t}=- & i \frac{\delta \mathcal{H}}{\delta a^{*}(\boldsymbol{k})} \\
= & -i \omega(\boldsymbol{k}) a(\boldsymbol{k}) \\
& -\frac{i}{2} \int V\left(\boldsymbol{k}, \boldsymbol{k}_{1}, \boldsymbol{k}_{2}\right) a\left(\boldsymbol{k}_{1}\right) a\left(\boldsymbol{k}_{2}\right) \delta\left(\boldsymbol{k}-\boldsymbol{k}_{1}-\boldsymbol{k}_{2}\right) d \boldsymbol{k}_{12} \\
& -i \int V^{*}\left(\boldsymbol{k}_{1}, \boldsymbol{k}, \boldsymbol{k}_{2}\right) a\left(\boldsymbol{k}_{1}\right) a^{*}\left(\boldsymbol{k}_{2}\right) \delta\left(\boldsymbol{k}_{1}-\boldsymbol{k}-\boldsymbol{k}_{2}\right) d \boldsymbol{k}_{12},
\end{aligned}
$$

$\omega(\boldsymbol{k})=k^{\alpha}, \quad V\left(\boldsymbol{k}, \boldsymbol{k}_{1}, \boldsymbol{k}_{2}\right)=\left(k k_{1} k_{2}\right)^{\beta}, \quad \alpha=3 / 2, \quad \beta=1 / 4$.

Here, $\boldsymbol{k}$ is a two-dimensional wave-number vector, $a^{*}$ expresses the complex conjugate of $a$, and c.c. also expresses the complex conjugate of the preceding term. The linear frequency and the complex amplitude of the mode of the wave number $\boldsymbol{k}$ are, respectively, expressed by $\omega(\boldsymbol{k})$ and $a(\boldsymbol{k})$. The shorthand notations $k=|\boldsymbol{k}|$ and $d \boldsymbol{k}_{12}=d \boldsymbol{k}_{1} d \boldsymbol{k}_{2}$ are used. When we derive the dynamic equations for the complex amplitude in weak turbulence systems, we often obtain a dynamic equation like Eq. (2a) when the three-wave resonant interactions are allowed like in the surface capillary waves [11]. In fact, the difference between the dynamic equation for surface capillary waves and that for our model appears only in the interaction kernel $V\left(\boldsymbol{k}, \boldsymbol{k}_{1}, \boldsymbol{k}_{2}\right)$. For our objective to generally compare the DNS with the RPA, we select the simple interaction kernel that allows the evaluation of the convolution in the nonlinear terms to be performed fast by fast Fourier transforms (FFT).
In numerical studies of weak turbulence, one sometimes adds artificial energy input and/or output to the conservative system (2a). If we were to investigate weak turbulence characteristics in a statistically steady state, such as the Kolmogorov-Zakharov spectrum, it would be necessary to add such nonconservative effects. On the other hand, the purpose of the present study is to assess the validity of the prediction of the RPA on the temporal evolution of various statistics of $a_{k}$. Therefore, we need statistical unsteadiness of the wave field, and in this respect the conservative system without input or output is in accordance with our purpose as it stands.

\section{B. Correspondence between continuous system and discrete system}

Some cautions should be exercised when we compare the theoretical description where the wave numbers are continuous with numerical results where the wave numbers are discrete. To connect the wave-number space and the real space, we select the definition of the Fourier transform as follows:

$$
f(\boldsymbol{x})=\frac{1}{2 \pi} \int F(\boldsymbol{k}) e^{i \boldsymbol{k} \cdot \boldsymbol{x}} d \boldsymbol{k}, \quad F(\boldsymbol{k})=\frac{1}{2 \pi} \int f(\boldsymbol{x}) e^{-i \boldsymbol{k} \cdot \boldsymbol{x}} d \boldsymbol{x},
$$

where $\boldsymbol{x}$ is a two-dimensional vector in the real space. We also select the Fourier series which connects the rectangular domain $R=L_{x} \times L_{y}$ in the real space under the doubly periodic boundary conditions to the discrete wave number $\boldsymbol{k}$ :

$$
f(\boldsymbol{x})=\sum_{\boldsymbol{k} \in S_{k}} F_{k} e^{i \boldsymbol{k} \cdot \boldsymbol{x}}, \quad F_{\boldsymbol{k}}=\frac{1}{L_{x} L_{y}} \int_{R} f(\boldsymbol{x}) e^{-i \boldsymbol{k} \cdot \boldsymbol{x}} d \boldsymbol{x},
$$

where $S_{\boldsymbol{k}}$ is a set of $\boldsymbol{k}$ allowed in the discrete wave-number space, i.e.,

$$
S_{\boldsymbol{k}}=\left\{\boldsymbol{k} \mid \boldsymbol{k}=\left(m \Delta k_{x}, n \Delta k_{y}\right),(m, n) \in \mathbb{Z}^{2}\right\} .
$$

The grid intervals in the discrete wave numbers $\Delta k_{x}$ and $\Delta k_{y}$ are connected to the periods in the real space $L_{x}$ and $L_{y}$ as

$$
\Delta k_{x}=\frac{2 \pi}{L_{x}}, \quad \Delta k_{y}=\frac{2 \pi}{L_{y}} .
$$

Because of the properties of the $\delta$ function,

$$
\delta(\boldsymbol{k})=\frac{1}{(2 \pi)^{2}} \int e^{i \boldsymbol{k} \cdot \boldsymbol{x}} d \boldsymbol{x},
$$

and of the Kronecker's $\delta$ for $\boldsymbol{k} \in S_{\boldsymbol{k}}$,

$$
\delta_{\boldsymbol{k}, \mathbf{0}}=\frac{1}{L_{x} L_{y}} \int_{R} e^{i \boldsymbol{k} \cdot \boldsymbol{x}} d \boldsymbol{x}=\frac{\Delta k_{x} \Delta k_{y}}{(2 \pi)^{2}} \int_{R} e^{i \boldsymbol{k} \cdot \boldsymbol{x}} d \boldsymbol{x},
$$

the correspondence

$$
\delta(\boldsymbol{k}) \longleftrightarrow \frac{1}{\Delta k_{x} \Delta k_{y}} \delta_{k, 0}
$$

is found for sufficiently small $\Delta k_{x}$ and $\Delta k_{y}$.

Because of the relation between $F(\boldsymbol{k})$ and $F_{\boldsymbol{k}}$ which generally holds,

$$
F(\boldsymbol{k})=\sum_{\boldsymbol{k}^{\prime} \in S_{\boldsymbol{k}}} 2 \pi F_{\boldsymbol{k}^{\prime}} \delta\left(\boldsymbol{k}-\boldsymbol{k}^{\prime}\right)
$$


and the correspondence (9), the following correspondence is found:

$$
a(\boldsymbol{k}) \longleftrightarrow \frac{2 \pi}{\Delta k_{x} \Delta k_{y}} a_{k},
$$

for $\boldsymbol{k} \in S_{\boldsymbol{k}}$.

The wave actions, $n(\boldsymbol{k})$ for the continuous system and $n_{k}$ for the discrete system, are respectively defined as

$$
n(\boldsymbol{k}) \delta\left(\boldsymbol{k}-\boldsymbol{k}^{\prime}\right)=\left\langle a(\boldsymbol{k}) a^{*}\left(\boldsymbol{k}^{\prime}\right)\right\rangle \text { and } n_{\boldsymbol{k}} \delta_{\boldsymbol{k}, \boldsymbol{k}^{\prime}}=\left\langle a_{\boldsymbol{k}} a_{\boldsymbol{k}^{\prime}}^{*}\right\rangle,
$$

where $\langle\cdots\rangle$ represents the ensemble average. The correspondences (9) and (11) give the correspondence of the wave actions:

$$
n(\boldsymbol{k}) \longleftrightarrow \frac{(2 \pi)^{2}}{\Delta k_{x} \Delta k_{y}} n_{\boldsymbol{k}} .
$$

The governing equation in the discretized wave numbers corresponding to Eq. (2a) is written as

$$
\begin{aligned}
\frac{d a_{k}}{d t}= & -i \omega_{\boldsymbol{k}} a_{\boldsymbol{k}}-\frac{i}{2}(2 \pi) \sum_{\boldsymbol{k}_{1}, \boldsymbol{k}_{2}} V_{\boldsymbol{k}_{1} \boldsymbol{k}_{2}}^{\boldsymbol{k}} a_{\boldsymbol{k}_{1}} a_{\boldsymbol{k}_{2}} \delta_{\boldsymbol{k}_{1} \boldsymbol{k}_{2}}^{k} \\
& -i(2 \pi) \sum_{\boldsymbol{k}_{1}, \boldsymbol{k}_{2}} V_{\boldsymbol{k} \boldsymbol{k}_{2}}^{\boldsymbol{k}_{1}} a_{\boldsymbol{k}_{1}} a_{\boldsymbol{k}_{2}}^{*} \delta_{\boldsymbol{k} \boldsymbol{k}_{2}}^{\boldsymbol{k}_{1}}
\end{aligned}
$$

where $\omega_{\boldsymbol{k}}=\omega(\boldsymbol{k}), V_{\boldsymbol{k}_{1} \boldsymbol{k}_{2}}^{\boldsymbol{k}}=V\left(\boldsymbol{k}, \boldsymbol{k}_{1}, \boldsymbol{k}_{2}\right)$, and $\delta_{\boldsymbol{k}_{1} \boldsymbol{k}_{2}}^{\boldsymbol{k}}$ expresses the Kronecker's $\delta, \delta_{\boldsymbol{k}, \boldsymbol{k}_{1}+\boldsymbol{k}_{2}}$. Note that, in addition to the fact that the integration and the $\delta$ function are, respectively, replaced by the summation and the Kronecker's $\delta$, the coefficients of the quadratic nonlinear terms are multiplied by $2 \pi$.

\section{Configuration of numerical simulations}

In our numerical simulations the wave-number space $\boldsymbol{k}=$ $\left(k_{x}, k_{y}\right)$ is discretized by the equally distributed grids with the interval $\Delta k=1 / 42$ in both $k_{x}$ and $k_{y}$ directions and is truncated along $\left|k_{x}\right|=k_{\max }$ and $\left|k_{y}\right|=k_{\max }$, with $k_{\max }=$ $512 \Delta k \approx 12$. The convolutions in the nonlinear terms are obtained by the pseudospectral transform method. In this method we first use inverse FFT of size $n_{x}=n_{y}=1024$ to transform $a_{k}$ to its inverse transform in the physical $\boldsymbol{x}$ space, perform there suitable multiplications, and then use the FFT to obtain the convolution sums. Although this pseudospectral transform method contains the aliasing error, the region $\left|k_{x}\right|,\left|k_{y}\right| \leqslant 341 \Delta k \approx 8$ in the $\boldsymbol{k}$ space is free from this aliasing error due to the $3 / 2$ rule. (For the $3 / 2$ rule, see, for example, Ref. [18].) We trace the temporal evolutions of $a_{k}$ only for those $\boldsymbol{k}$ 's which are within this alias-free region.

By reference to the Pierson-Moskowitz spectrum that is typical in the ocean waves, we employ an isotropic spectrum as follows for the initial wave field:

$$
\begin{aligned}
H_{2} & =\sum_{k} \omega_{k}\left|a_{k}\right|^{2}, \\
\left|a_{k}\right|^{2} & =A k^{-6.5} \exp \left(-1 / k^{4}\right) D(k), \\
D(k) & = \begin{cases}1, & (0<k<7), \\
\exp \left(-10(k-7)^{2}\right), & (7 \leqslant k \leqslant 8),\end{cases}
\end{aligned}
$$

where $\mathrm{H}_{2}$ is the discrete counterpart of the lowest-order Hamiltonian $\mathcal{H}_{2}$ of Eq. (1b). The exponential function and the power-law function in Eq. (15b), respectively, give the increase in the small wave numbers and the decrease in the large wave numbers. The function $D(k)$ gives the exponential tail near the end of the alias-free wave numbers so that the truncation in the $\boldsymbol{k}$ space does not affect the numerical results. For the purpose of this study the choice of the spectrum is arbitrary, and this spectrum (15) does not have any special significance for the system at all. Here, the coefficient $A$ is a parameter to control the value of $\mathrm{H}_{2}$. In this study, we performed four series of simulations which have $H_{2}=1.25 \times 10^{-6}, 2.5 \times 10^{-6}$, $5 \times 10^{-6}$, and $1 \times 10^{-5}$. The initial phases of each component wave are given by uniform random numbers in the range $[0,2 \pi]$. The ratio $\left|H_{3} / H_{2}\right|$ can be a measure of the degree of nonlinearity of the wave field as a whole, where $\mathrm{H}_{3}$ is the discrete counterpart of the interaction Hamiltonian $\mathcal{H}_{3}$ of Eq. (1c). $\left|H_{3} / H_{2}\right|$ is an increasing function of $H_{2}$ and takes values around $5.0 \times 10^{-5}$, when $H_{2}=1.25 \times 10^{-6}$, and $3.5 \times 10^{-4}$, when $H_{2}=1 \times 10^{-5}$. Our selection of the values of $H_{2}$ as above is made to keep $\left|H_{3} / H_{2}\right|$ and hence the nonlinearity of the wave field sufficiently small. To make the ensemble average, 256 independent simulations which have different initial phases are performed for each $H_{2}$. The time integration is made until $t=100 T_{p}$. Here, $T_{p}=2 \pi$ is the period given by the linear dispersion relation for $k=1$, at which the one-dimensional energy spectrum defined below has its maximum. The fourth-order Runge-Kutta method with a time interval of $\Delta t=T_{p} / 50$ is employed for the time integration. The linear term is implicitly solved to improve the numerical stability. Because the system and the initial spectrum are isotropic, $a_{k}$ for $k>8$ is set to 0 at each time step.

The total Hamiltonian that is the sum of the linear part $\mathrm{H}_{2}$ and the nonlinear part $\mathrm{H}_{3}$ is numerically conserved within the relative error $2.6 \times 10^{-4}$ for $H_{2}=1 \times 10^{-5}$, where the nonlinearity is the largest and the conservation is the worst. In this case, the average of $H_{3}$ during $100 T_{p}$ is $-3.5 \times 10^{-9}$.

All the numerical simulations are performed on the FUJITSU FX1 at the Information Technology Center, Nagoya University. The CPU time for one realization takes $12 \mathrm{~h}$.

\section{NUMERICAL RESULTS}

\section{A. Time evolution of spectra}

Figure 1 shows the azimuthally-integrated one-dimensional energy spectra $E(k)$

$$
E(k)=\frac{1}{\Delta_{\text {bin }}} \sum_{k-\Delta_{\text {bin }} / 2<\left|\boldsymbol{k}^{\prime}\right|<k+\Delta_{\text {bin }} / 2}\left\langle\omega_{\boldsymbol{k}^{\prime}}\left|a_{\boldsymbol{k}^{\prime}}\right|^{2}\right\rangle,
$$

obtained from DNS at $t=100 T_{p}$. The initial spectra are also shown for reference. Here, $\Delta_{\text {bin }}$ denotes the width of the bins that is used to evaluate $E(k)$ from the complex amplitudes defined on the discrete wave numbers, and we set $\Delta_{\text {bin }}=0.05$. Figures 1(a), 1(b), 1(c), and 1(d) show the energy spectra for $H_{2}=1.25 \times 10^{-6}, H_{2}=2.5 \times 10^{-6}, H_{2}=5 \times 10^{-6}$, and $H_{2}=1 \times 10^{-5}$, respectively.

Small irregularities around $k=1.1$ are due to the numerical procedures to obtain $E(k)$ in the discrete $\boldsymbol{k}$ space. While the variation of the spectrum during $100 T_{p}$ for $H_{2}=1.25 \times 10^{-6}$ is quite small, that for $H_{2}=1 \times 10^{-5}$ is large during the 
(a)

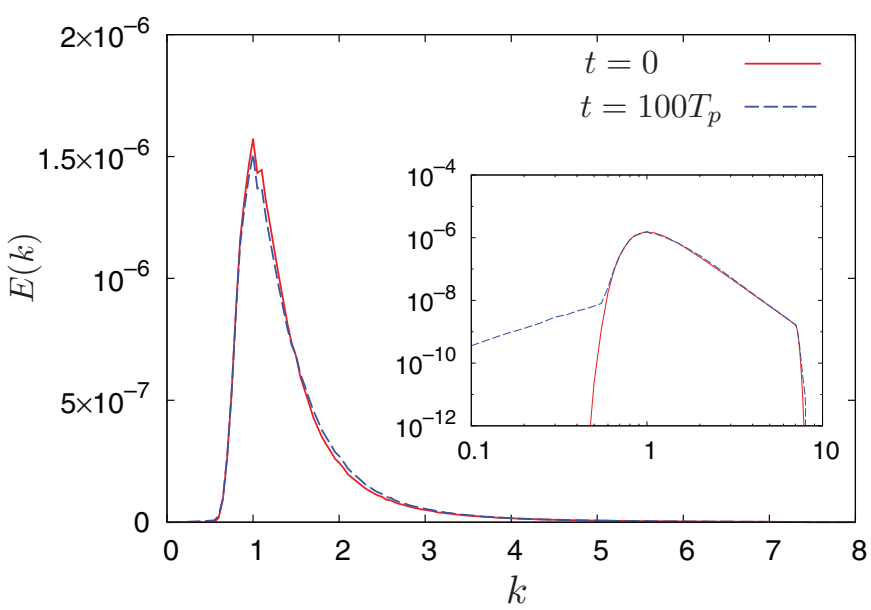

(c)

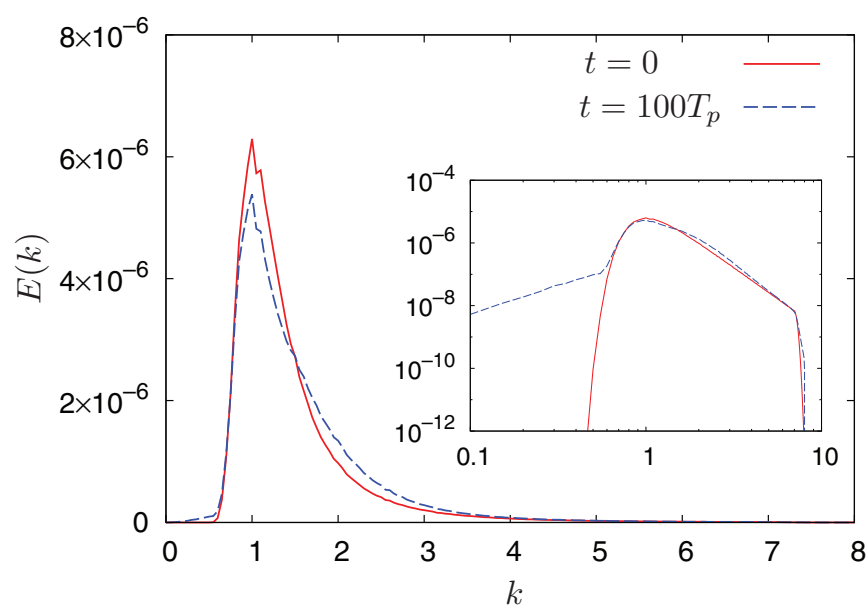

(b)

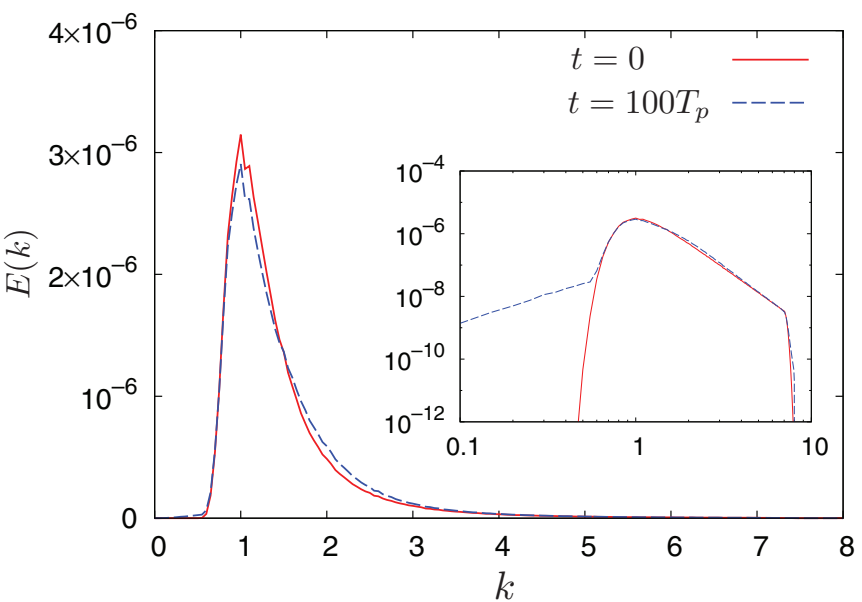

(d)

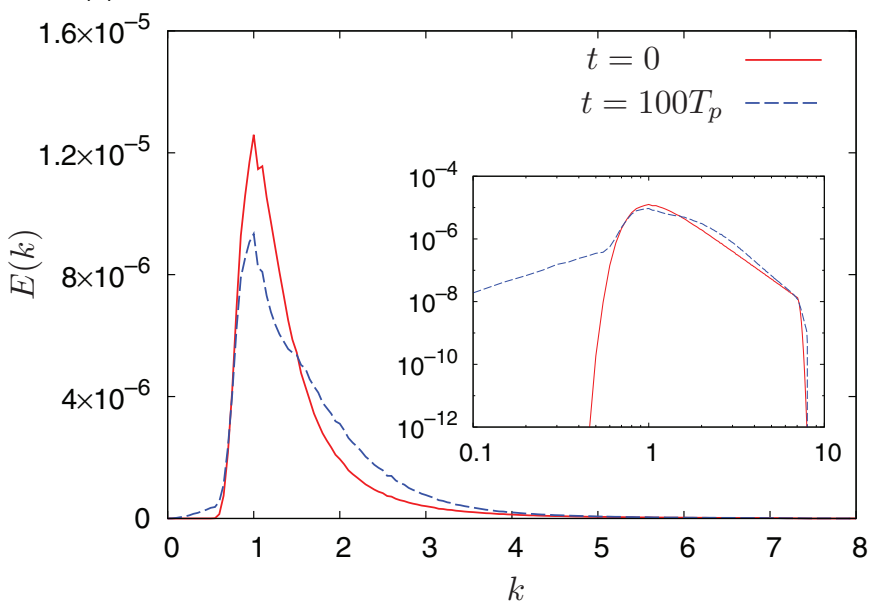

FIG. 1. (Color online) Variations of one-dimensional energy spectra $E(k)$ from $t=0$ to $t=100 T_{p}$. The spectra are shown with the double logarithmic scales in the insets. (a) $H_{2}=1.25 \times 10^{-6}$, (b) $H_{2}=2.5 \times 10^{-6}$, (c) $H_{2}=5 \times 10^{-6}$, and (d) $H_{2}=1 \times 10^{-5}$.

time. Most of the weak turbulence theories, including the RPA studied here, have been developed for weak turbulence, where the linear time scale determined by the linear frequency and the nonlinear time scale, i.e., the time scale of spectral change, are largely separated. Therefore, the weakly nonlinear assumption might be slightly violated for $H_{2}=1 \times 10^{-5}$, although the assumption is evidently valid for $H_{2}=1.25 \times 10^{-6}$.

The wave action $n(\boldsymbol{k})$ of the three-wave system (2) is known to evolve according to the following kinetic equation (see, for example, Refs. [11,13]):

$$
\begin{aligned}
\frac{d n(\boldsymbol{k})}{d t}= & -\gamma(\boldsymbol{k}) n(\boldsymbol{k})+\eta(\boldsymbol{k}), \\
\eta(\boldsymbol{k})= & \pi \int\left\{\left|V\left(\boldsymbol{k}, \boldsymbol{k}_{1}, \boldsymbol{k}_{2}\right)\right|^{2} \delta\left(\boldsymbol{k}-\boldsymbol{k}_{1}-\boldsymbol{k}_{2}\right) \delta\left(\omega(\boldsymbol{k})-\omega\left(\boldsymbol{k}_{1}\right)-\omega\left(\boldsymbol{k}_{2}\right)\right)\right. \\
& \left.+2\left|V\left(\boldsymbol{k}_{2}, \boldsymbol{k}, \boldsymbol{k}_{1}\right)\right|^{2} \delta\left(\boldsymbol{k}_{2}-\boldsymbol{k}-\boldsymbol{k}_{1}\right) \delta\left(\omega\left(\boldsymbol{k}_{2}\right)-\omega(\boldsymbol{k})-\omega\left(\boldsymbol{k}_{1}\right)\right)\right\} n_{\boldsymbol{k}_{1}} n_{\boldsymbol{k}_{2}} d \boldsymbol{k}_{12}, \\
\gamma(\boldsymbol{k})= & 2 \pi \int\left\{\left|V\left(\boldsymbol{k}, \boldsymbol{k}_{1}, \boldsymbol{k}_{2}\right)\right|^{2} \delta\left(\boldsymbol{k}-\boldsymbol{k}_{1}-\boldsymbol{k}_{2}\right) \delta\left(\omega(\boldsymbol{k})-\omega\left(\boldsymbol{k}_{1}\right)-\omega\left(\boldsymbol{k}_{2}\right)\right) n\left(\boldsymbol{k}_{2}\right)\right. \\
& \left.+\left|V\left(\boldsymbol{k}_{2}, \boldsymbol{k}, \boldsymbol{k}_{1}\right)\right|^{2} \delta\left(\boldsymbol{k}_{2}-\boldsymbol{k}-\boldsymbol{k}_{1}\right) \delta\left(\omega\left(\boldsymbol{k}_{2}\right)-\omega(\boldsymbol{k})-\omega\left(\boldsymbol{k}_{1}\right)\right)\left[n\left(\boldsymbol{k}_{2}\right)-n\left(\boldsymbol{k}_{1}\right)\right]\right\} d \boldsymbol{k}_{12} .
\end{aligned}
$$

Since the energy spectrum for $H_{2}=1.25 \times 10^{-6}$ varies very little during the time $100 T_{p}$ as shown in Fig. 1(a), the right-hand side of Eq. (17a) is almost constant in time and the wave action is expected to be a linear function of time. Figure 2 shows the time rates of change of $E(k)$ for $H_{2}=1.25 \times 10^{-6}$. One is obtained for the initial spectrum (15) according to Eq. (17). The other is obtained from DNS as the difference between the energy spectrum at $t=50 T_{p}$ and that at $t=0$ divided by $50 T_{p}$. Both time rates of change agree quite well. 


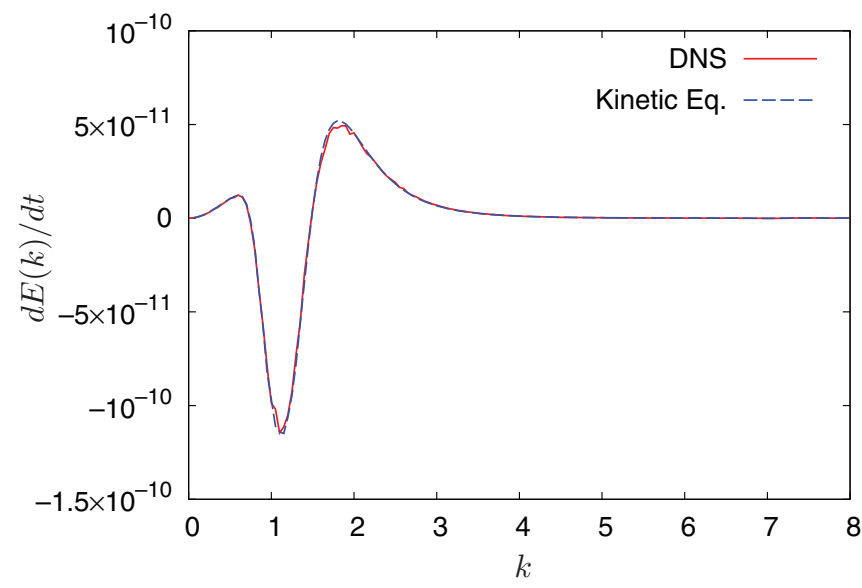

FIG. 2. (Color online) Comparison of the time rates of change of $E(k)$ between the DNS and the kinetic equation. $H_{2}=1.25 \times 10^{-6}$.

The same procedure of the comparison cannot be used for $H_{2}=1 \times 10^{-5}$, since the variation of $E(k)$ is large. Then, the energy spectrum at $100 T_{p}$ is obtained by the numerical integration of Eq. (17) in time, and it is compared with the energy spectrum at $t=100 T_{p}$ obtained by DNS, which is already shown in Fig. 1(d). The comparison is shown in Fig. 3. Also for $H_{2}=1 \times 10^{-5}$, both spectra agree quite well. It clearly shows that the spectrum in DNS evolves in time according to the prediction of the kinetic equation (17).

\section{B. Importance of resonant interaction for evolution of fluctuation}

In our DNS, the initial value of the amplitude of each wave mode $\left|a_{k}(0)\right|$ is determined by the initial spectrum; hence no amplitude fluctuation exists at $t=0$. The fluctuation grows as time elapses through nonlinear interactions with other modes. Examples of the evolution of $\left|a_{k}\right|^{2}$ are shown in Fig. 4. The five curves show the variations of $\left|a_{k}\right|^{2}$ for $\boldsymbol{k}=(3,0)$ in five independent realizations for $H_{2}=5 \times 10^{-6}$. Since the simulations are started without the amplitude fluctuations, all

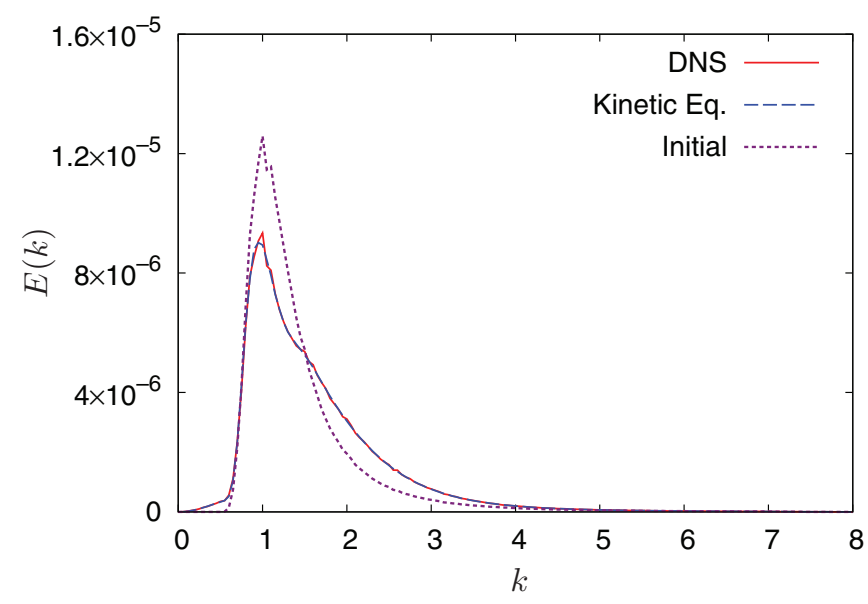

FIG. 3. (Color online) Comparison of the variations of $E(k)$ from $t=0$ to $t=100 T_{p}$ between the DNS and the kinetic equation. $H_{2}=$ $1 \times 10^{-5}$.

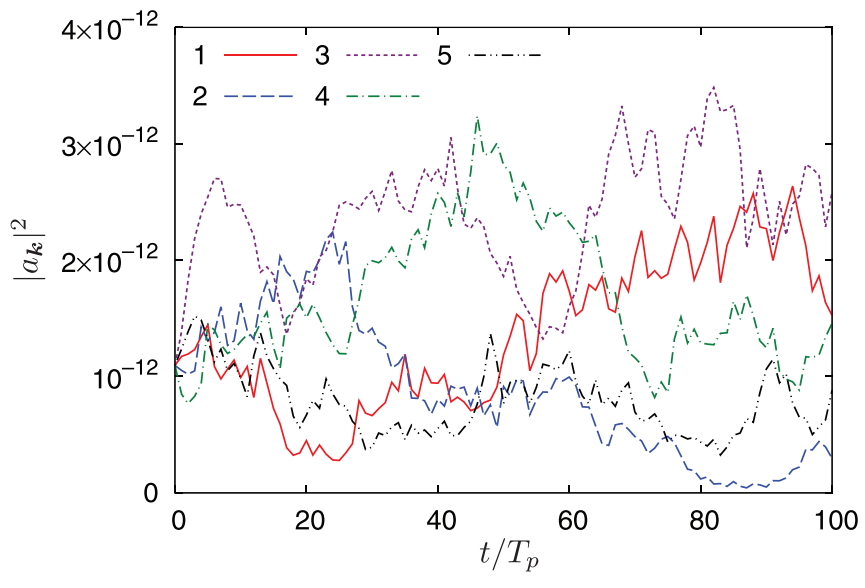

FIG. 4. (Color online) Evolution of $\left|a_{k}\right|^{2}$ in five independent realizations. $H_{2}=5 \times 10^{-6}, \boldsymbol{k}=(3,0)$.

$\left|a_{k}\right|^{2}$ has the same value at $t=0$. The fluctuations grow as time elapses, and each $\left|a_{k}\right|^{2}$ evolves differently.

Figure 5 shows the time evolutions of the mean $n_{k}$ and the standard deviation $\sigma_{k}$ of $\left|a_{k}\right|^{2}$ obtained from DNS for $\mathrm{H}_{2}=$ $5 \times 10^{-6}$. Figures 5(a), 5(b), $5(\mathrm{c})$, and 5(d), respectively, show the evolution of $n_{k}$ and $\sigma_{k}$ of $k=1, k=1.5, k=3$, and $k=6$. Throughout this paper, to evaluate the statistical quantities at $k$ such as $n_{k}$, the quantity is averaged over the modes of $\boldsymbol{k}^{\prime}$ in the annular domain $\left|k-k^{\prime}\right|<\Delta_{\text {bin }} / 2(=0.025)$. It can be seen that $\sigma_{k}$, that is, the amplitude fluctuation, grows in time at each $k$, although the growth rates are wave number dependent. In particular, at the larger wave numbers $k=3$ and $k=6$, it is clearly observed that the fluctuations are approaching the Gaussianity of $a_{k}$, i.e., $n_{k}=\sigma_{k}$.

For comparison, we performed another series of DNS where the power-law exponent $\alpha$ of the linear dispersion relation in Eq. (2b) is changed to $\alpha=1 / 2$ while all the other aspects of the model remain intact. In this case, the dispersion relation $\omega=$ $k^{1 / 2}$ is of nondecay type, which is similar to the surface gravity waves in water, and the three-wave resonant interactions are prohibited. The results are shown in Fig. 6 where we draw the same quantities shown in Figs. 4 and 5(c). By comparing Fig. 6 with Figs. 4 and 5(c), we observe that the growth of the fluctuations is much slower when the three-wave resonant interactions are prohibited by the dispersion relation. Similarly, the slower growths are observed for other wave numbers.

The kinetic equation (17) derived by the weak turbulence theory represents that only the resonant interactions play a role in the evolution of the wave action $n_{k}$. It can simply be understood since the secular energy transfer persistent against the time average is necessary for $n_{k}$, which is the mean of $\left|a_{k}\right|^{2}$, to evolve and only the resonant interactions can provide it. On the other hand, the fluctuations of $\left|a_{k}\right|^{2}$ around its mean could grow owing to the nonresonant nonlinear interactions as well as the resonant ones, because the nonresonant interactions seem to work as the stochastic driving forces in random walk processes. However, comparison between Figs. 4 and 5 and Fig. 6 clearly shows that the resonant interactions are essential for the evolution of the fluctuations. Even though the three-wave resonances are prohibited when $\alpha=1 / 2$ and our model (2a) contains only quadratic nonlinear terms, the 
(a)

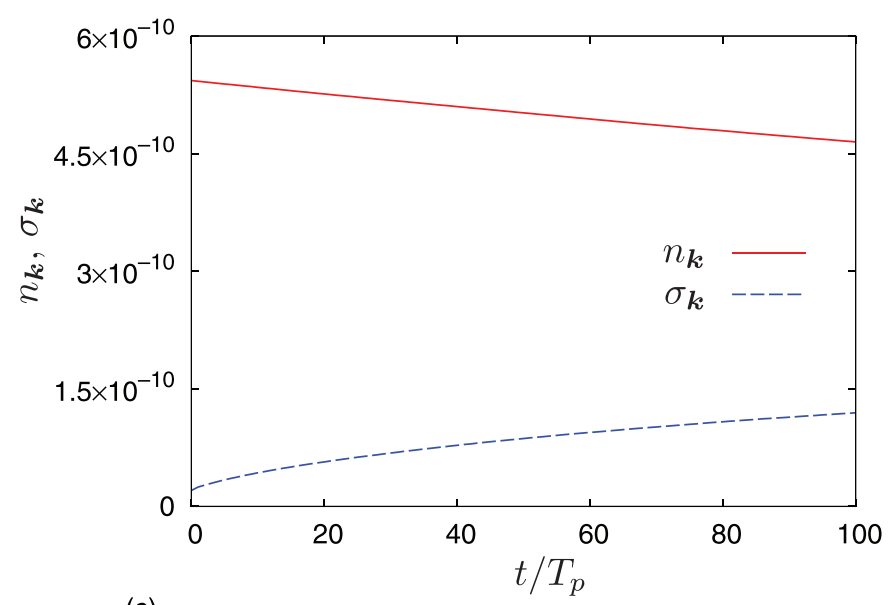

(c)

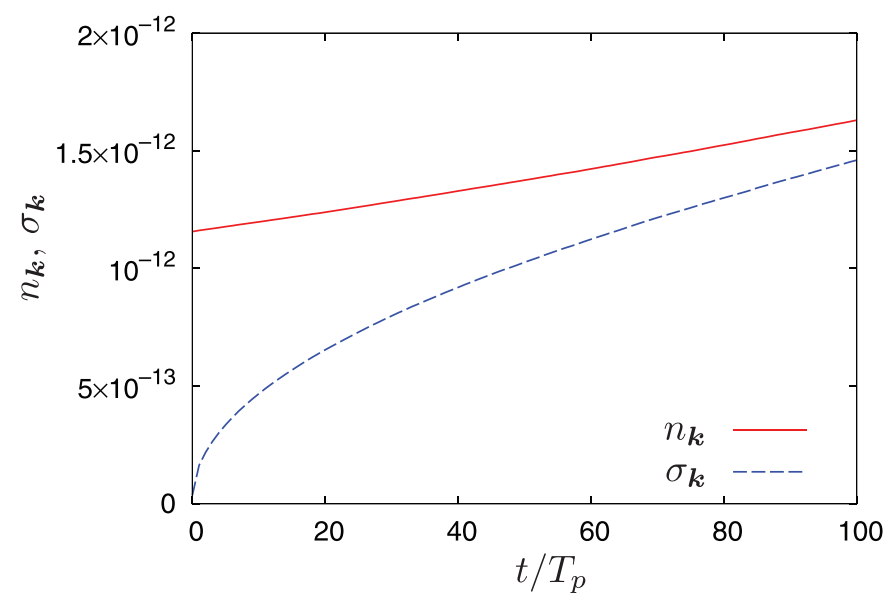

(b)

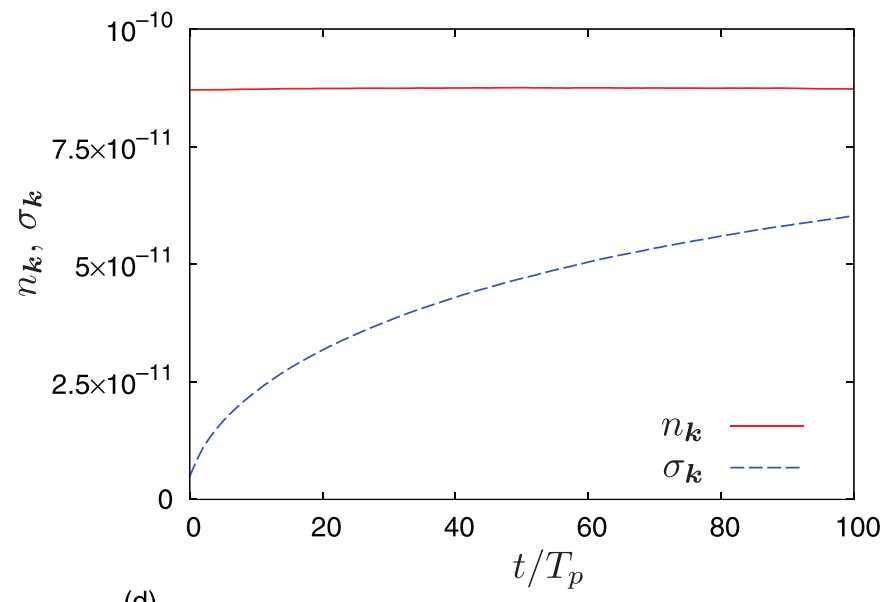

(d)

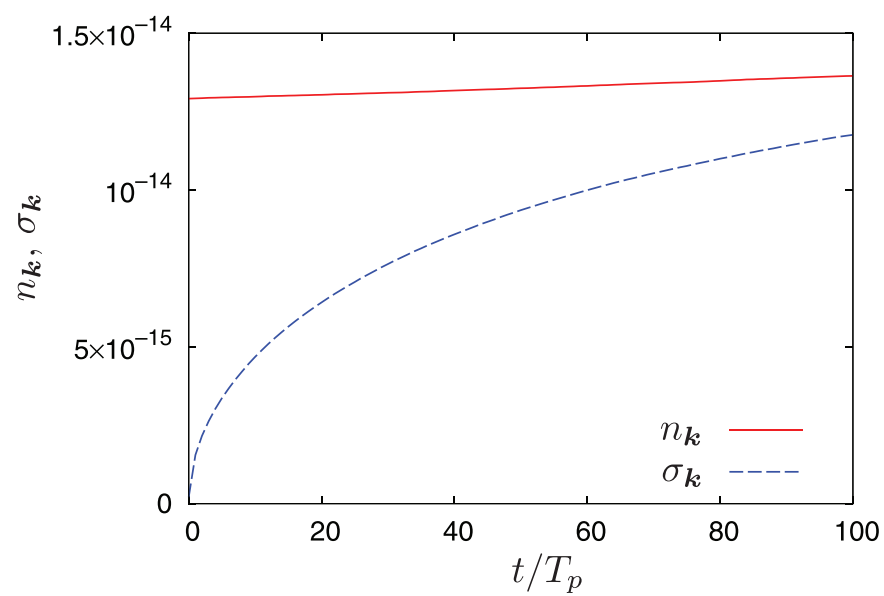

FIG. 5. (Color online) Evolution of the means $n_{k}$ and the standard deviations $\sigma_{k}$ of $\left|a_{k}\right|^{2} . H_{2}=5 \times 10^{-6}$. (a) $k=1$, (b) $k=1.5$, (c) $k=3$, and (d) $k=6$.

fluctuations do grow as shown in Fig. 6(b) albeit very slowly. This is because two nonresonant three-wave interactions can make a resonant four-wave interaction, and hence the fluctuations grow due to this four-wave resonant interaction with a much slower time scale.

\section{Approach to Gaussianity}

The $p$ th-order moment is defined as

$$
M^{(p)}(\boldsymbol{k})=\left\langle\left(\frac{|a(\boldsymbol{k})|^{2}}{\delta(\mathbf{0})}\right)^{p}\right\rangle,
$$

where $\delta(\mathbf{0})$ is defined in the large-box limit as

$$
\delta(\mathbf{0})=\lim _{L_{x}, L_{y} \rightarrow \infty} \frac{L_{x} L_{y}}{(2 \pi)^{2}}=\lim _{\Delta k_{x}, \Delta k_{y} \rightarrow 0} \frac{1}{\Delta k_{x} \Delta k_{y}},
$$

according to the correspondence (9). The RPA predicts that $M^{(p)}(\boldsymbol{k})$ evolves according to the following equation [14]:

$$
\frac{d M^{(p)}(\boldsymbol{k})}{d t}=-p \gamma(\boldsymbol{k}) M^{(p)}(\boldsymbol{k})+p^{2} \eta(\boldsymbol{k}) M^{(p-1)}(\boldsymbol{k}),
$$

where $\eta(\boldsymbol{k})$ and $\gamma(\boldsymbol{k})$ are, respectively, given in Eqs. (17b) and (17c). Equation (20) for $p=1$ is identical to the kinetic equation (17) for the wave action $n(\boldsymbol{k})$.
When the real and imaginary parts of $a(\boldsymbol{k})$ are independent and obey the same Gaussian distribution,

$$
M^{(p)}(\boldsymbol{k})=p ! n^{p}(\boldsymbol{k}) .
$$

Then,

$$
F^{(p)}(\boldsymbol{k})=\frac{M^{(p)}(\boldsymbol{k})-p ! n^{p}(\boldsymbol{k})}{p ! n^{p}(\boldsymbol{k})},
$$

is an index which expresses the deviation from the Gaussianity of $a(\boldsymbol{k})$. Equation (20) can be written for $F^{(p)}(\boldsymbol{k})$ as

$$
\frac{d F^{(p)}(\boldsymbol{k})}{d t}=\frac{p \eta(\boldsymbol{k})}{n(\boldsymbol{k})}\left[F^{(p-1)}(\boldsymbol{k})-F^{(p)}(\boldsymbol{k})\right] .
$$

Because $F^{(1)}(\boldsymbol{k})=0$ by the definition that $M^{(1)}(\boldsymbol{k})=n(\boldsymbol{k})$, the equation for the evolution of $F^{(2)}(\boldsymbol{k})$ can be obtained as

$$
\frac{d F^{(2)}(\boldsymbol{k})}{d t}=-\frac{2 \eta(\boldsymbol{k})}{n(\boldsymbol{k})} F^{(2)}(\boldsymbol{k})
$$

When $|a(\boldsymbol{k})|$ is deterministically given and no fluctuation is allowed like in the initial conditions of our DNS, $F^{(2)}(\boldsymbol{k})=$ $-1 / 2$. Equation (23) represents that the deviation from the Gaussianity of $a(\boldsymbol{k})$ for all $p$ decays after an elapse of sufficient time, because $\eta(\boldsymbol{k})>0$. The homogeneous term in Eq. (23) 
(a)

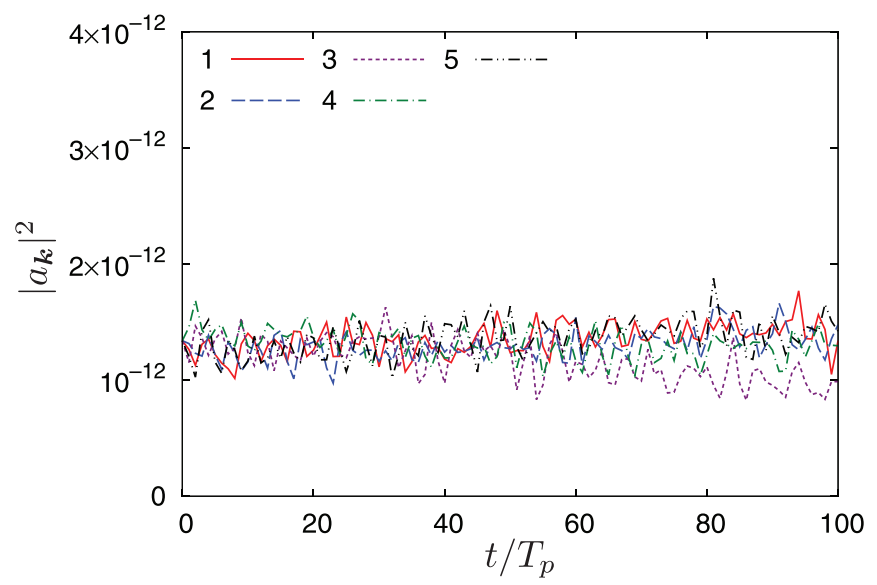

(b)

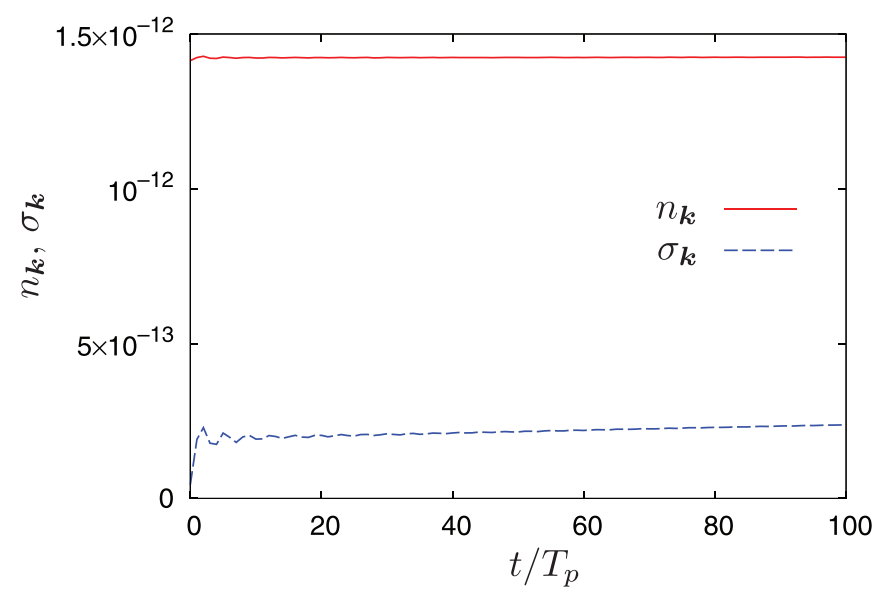

FIG. 6. (Color online) Evolution of the fluctuations of $\left|a_{k}\right|^{2} . \omega=$ $k^{1 / 2}$ and $H_{2}=5 \times 10^{-6}$. (a) $\left|a_{k}\right|^{2}$ in five independent realizations. $\boldsymbol{k}=(3,0)$. (b) The mean $n_{k}$ and the standard deviation $\sigma_{k}$ of $\left|a_{k}\right|^{2}$. $k=3$.

indicates that $F^{(p)}(\boldsymbol{k})$ for large $p$ decays fast, and then the nonhomogeneous term of the order $p-1$ can be dominant in the evolution of $F^{(p)}(\boldsymbol{k})$. Therefore, the evolution of $F^{(p)}(\boldsymbol{k})$ for all $p$ is determined by the slowest $F^{(2)}(\boldsymbol{k})$.

According to Eqs. (23) and (24), the speed at which $a(\boldsymbol{k})$ approaches the Gaussianity depends on the value of $\eta(\boldsymbol{k}) / n(\boldsymbol{k})$. Figure 7(a) shows the evolution of $\eta(\boldsymbol{k}) / n(\boldsymbol{k})$ for $k=1,1.5,3$, and 6 for $H_{2}=5 \times 10^{-6}$, and Fig. 7(b) shows the time average, the maximum and the minimum of $\eta(\boldsymbol{k}) / n(\boldsymbol{k})$ at each $k$ during $100 T_{p}$. These results are obtained by the numerical integration of the kinetic equation (17). For the initial spectrum (15c), $\eta(\boldsymbol{k}) / n(\boldsymbol{k})$ is large, in the range $2 \lesssim k \lesssim 3$. The wave-number dependence of $\eta(\boldsymbol{k}) / n(\boldsymbol{k})$ is consistent with the rapid growth of the fluctuation at $k=3$ compared with those at the other wave numbers observed in Fig. 5.

As shown in Fig. 1, the spectral variation for $H_{2}=5 \times$ $10^{-6}$ during $100 T_{p}$ is not small. However, Fig. 7(b) indicates that the variation of the value of $\eta(\boldsymbol{k}) / n(\boldsymbol{k})$ during $100 T_{p}$ is almost constant in time except $1.5<k<3$, because the differences between the maximums and the minimums are small. Then, Eq. (24) suggests that $F^{(2)}(\boldsymbol{k})$ shows exponential decay in time. Figure 8 shows the evolution of $F^{(2)}$ of $k=3$ (a)

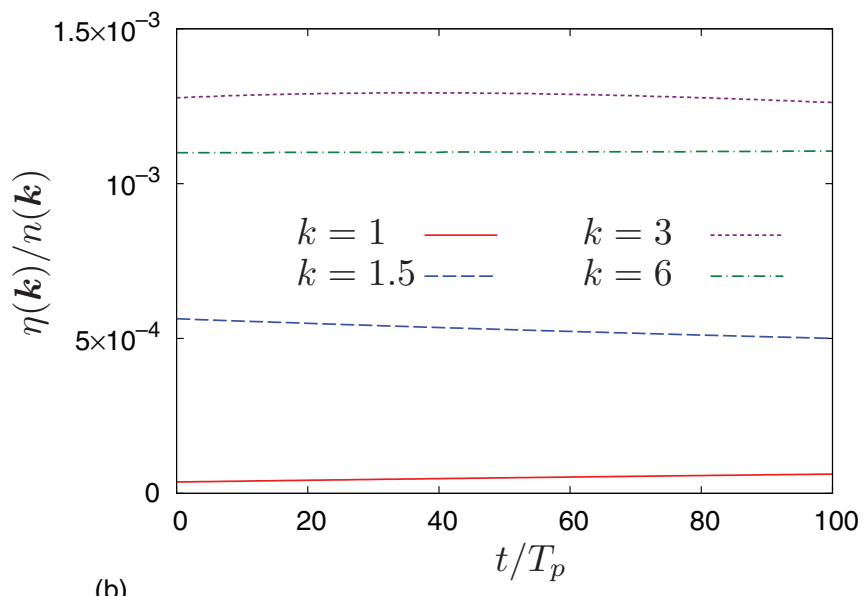

(b)

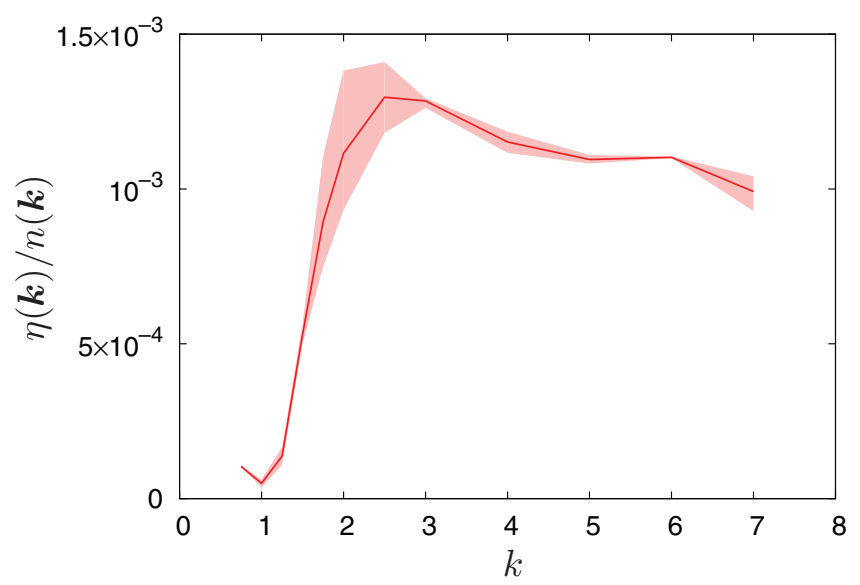

FIG. 7. (Color online) Value of $\eta(\boldsymbol{k}) / n(\boldsymbol{k})$ for each wave number. $H_{2}=5 \times 10^{-6}$. (a) Evolution. (b) Time average, maximum and minimum during $0 \leqslant t \leqslant 100 T_{p}$.

for $H_{2}=5 \times 10^{-6}$ in DNS. It is found that the absolute value of $F^{(2)}$ exponentially decays as expected. When $F^{(2)}$ is fitted by an exponential function $-1 / 2 \exp (-\lambda(\boldsymbol{k}) t)$, the decay rate $\lambda(\boldsymbol{k})$ obtained by the method of least squares is $2.58 \times 10^{-3}$. On the other hand, the decay rate predicted by the RPA is

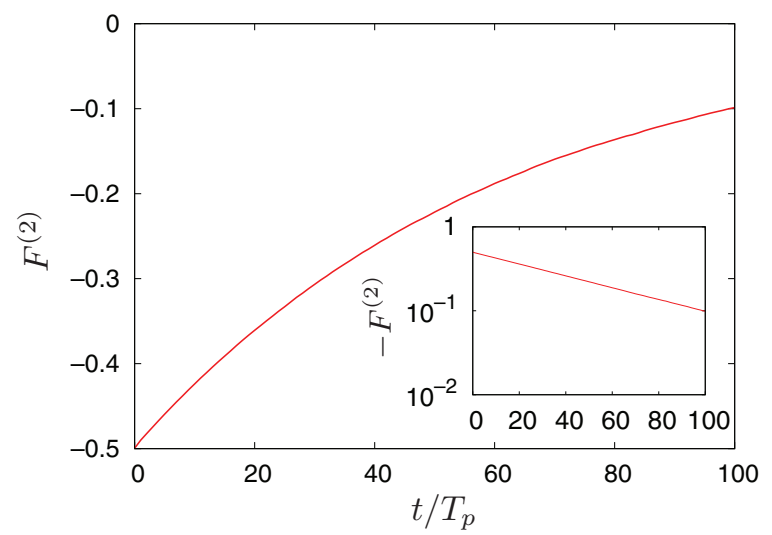

FIG. 8. (Color online) Evolution of $F^{(2)}$ in DNS. $H_{2}=5 \times 10^{-6}$, $k=3$. The evolution is shown with the single logarithmic scale in the inset. 


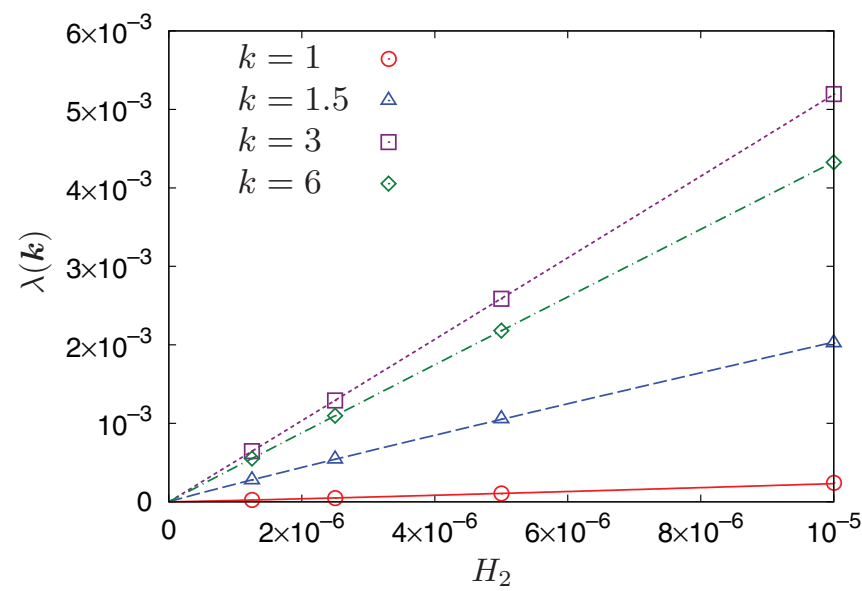

FIG. 9. (Color online) Dependence of the decay rate $\lambda(\boldsymbol{k})$ of $F^{(2)}(\boldsymbol{k})$ in DNS on $\mathrm{H}_{2}$.

$2 \eta(\boldsymbol{k}) / n(\boldsymbol{k})$. The time average of $2 \eta(\boldsymbol{k}) / n(\boldsymbol{k})$ during $100 T_{p}$ is $2.57 \times 10^{-3}$ for $k=3$. Thus the quantitative agreement between the RPA and the DNS is quite good.

Figure 9 shows the decay rate $\lambda(\boldsymbol{k})$ for $k=1,1.5,3$, and 6 obtained by the least-square fit of the exponential function to $F^{(2)}(\boldsymbol{k})$ in DNS as a function of $H_{2}$. It is observed that $\lambda(\boldsymbol{k})$ for each wave number is proportional to $H_{2}$. As shown by Eqs. (17) and (24), the RPA predicts that the time scales of the growth of the fluctuations as well as those of the evolution of the spectra are inversely proportional to the value of the Hamiltonian. It is consistent with the results shown in Fig. 9.

\section{Moments for $p>2$}

The solution of Eq. (23) is given as

$$
\begin{aligned}
F^{(p)}(\boldsymbol{k}, t) & =\sum_{j=2}^{p} C_{j}^{(p)} e^{-j \theta(\boldsymbol{k})}, \\
\theta(\boldsymbol{k}) & =\int_{0}^{t} \frac{\eta\left(\boldsymbol{k}, t^{\prime}\right)}{n\left(\boldsymbol{k}, t^{\prime}\right)} d t^{\prime} .
\end{aligned}
$$

The coefficient $C_{j}^{(p)}$ is determined by the recurrence formula

$$
\begin{aligned}
& C_{j}^{(p)}=\left(\begin{array}{c}
p \\
j
\end{array}\right) C_{j}^{(j)} \quad(j=2, \ldots, p-1), \\
& C_{2}^{(2)}=F^{(2)}(\boldsymbol{k}, 0), \quad C_{p}^{(p)}=F^{(p)}(\boldsymbol{k}, 0)-\sum_{j=2}^{p-1} C_{j}^{(p)},
\end{aligned}
$$

where $\left({ }_{j}^{p}\right)$ is the binomial coefficient. ${ }^{1}$

The deviations from the Gaussianity $F^{(p)}$ at $k=7$ at every $20 T_{p}$ from $t=0$ to $t=100 T_{p}$ for $H_{2}=5 \times 10^{-6}$ obtained by Eq. (25) and DNS are shown in Fig. 10 as functions of the order $p$ of the moments. A large number of $a_{k}$ are required to obtain reliable high-order moments. As explained before, to evaluate statistical quantities such as the moments of $a_{k}$ at $k$, we use the values of $a_{k^{\prime}}$ in the annular domain $\left|k^{\prime}-k\right|<0.025$;

\footnotetext{
${ }^{1}$ The explicit expression of the solution of Eq. (23) in Ref. [12] is incorrect.
}

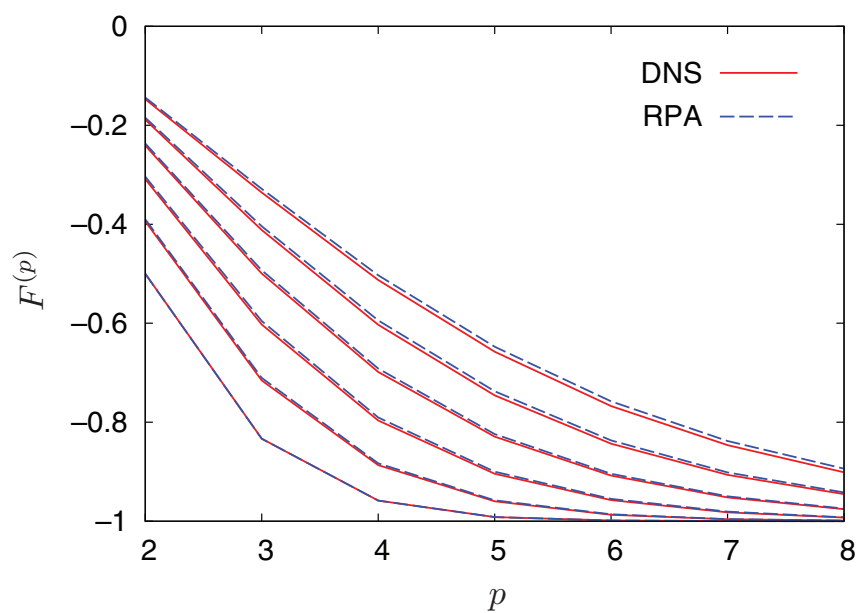

FIG. 10. (Color online) Comparison of $F^{(p)}$ between the DNS and the RPA for each $p . t=0,20 T_{p}, \ldots, 100 T_{p}$ from bottom to top. $H_{2}=5 \times 10^{-6}$ and $k=7$.

hence the number of modes used in the statistical evaluation increases in proportion to $k$. This gives the reason to choose the large wave number $k=7$ to evaluate the high-order moments. We confirmed that the tail of the probability density function of $\left|a_{k}\right|^{2}$ defined below decays faster than the negative nineth power of $\left|a_{k}\right|^{2}$. Therefore, the moments up to the eighth order are reliable. To obtain the deviation $F^{(p)}$ by Eq. (25) in the RPA, $\theta(\boldsymbol{k})$ is approximated by $\theta(\boldsymbol{k}) \approx \overline{(\eta / n)} t$, where $\overline{(\eta / n)}$ is the time-averaged value of $\eta(\boldsymbol{k}) / n(\boldsymbol{k})$ during $100 T_{p}$. Similarly, the evolution of the deviations $F^{(p)}$ at $k=7$ obtained by the DNS and the RPA are compared in Fig. 11. Even though the value of $\theta(\boldsymbol{k})$ is approximated by $\overline{(\eta / n)} t$ and the high-order moments such as those of the eighth-order are treated, the RPA and the DNS show another good agreement.

\section{E. Evolution of distribution of amplitude fluctuations}

The RPA also gives the evolution of the PDF $\mathcal{P}(s(\boldsymbol{k}))$ of $s(\boldsymbol{k})$, which is a stochastic variable defined by

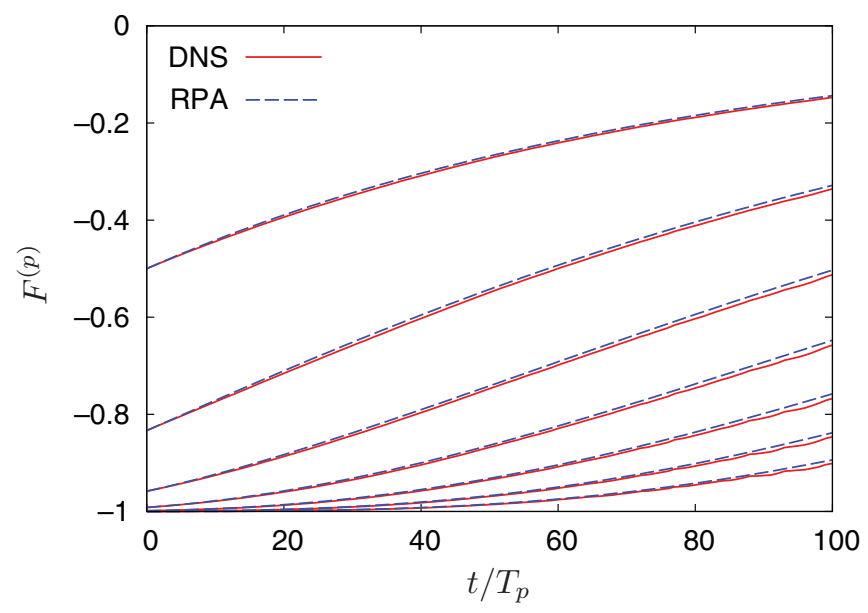

FIG. 11. (Color online) Evolution of $F^{(p)}$ in the DNS and the RPA. $p=2,3, \ldots, 8$ from top to bottom. $H_{2}=5 \times 10^{-6}, k=7$. 


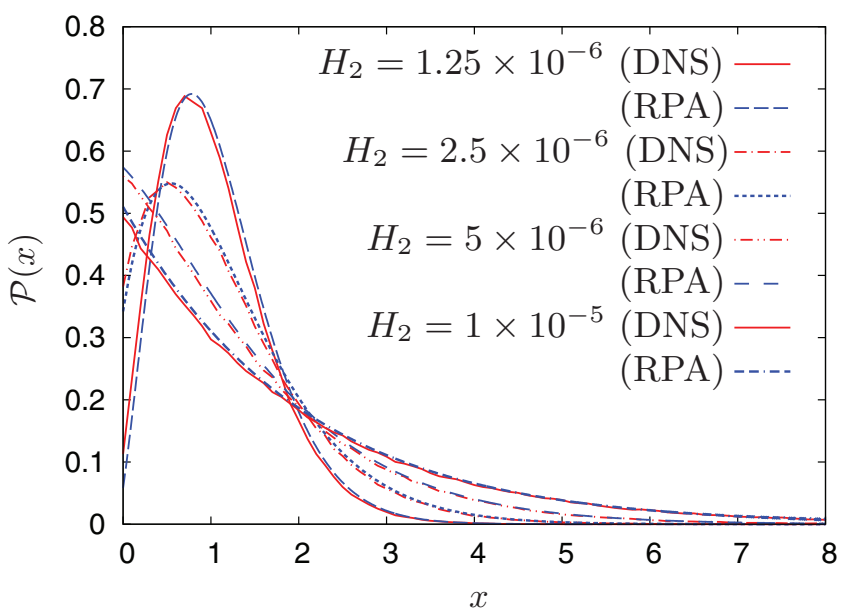

FIG. 12. (Color online) Comparison of $\mathcal{P}(x)$ in the DNS and the RPA. $k=3$ and $t=100 T_{p}$.

$$
\begin{aligned}
s(\boldsymbol{k})=|a(\boldsymbol{k})|^{2} / \delta(\boldsymbol{0}), \text { as follows [14]: } & \begin{aligned}
\frac{\partial \mathcal{P}(s(\boldsymbol{k}))}{\partial t} & =\frac{\partial \mathcal{F}(\boldsymbol{k})}{\partial s(\boldsymbol{k})}, \\
\mathcal{F}(\boldsymbol{k}) & =s(\boldsymbol{k})\left\{\gamma(\boldsymbol{k}) \mathcal{P}(s(\boldsymbol{k}))+\eta(\boldsymbol{k}) \frac{\partial \mathcal{P}(s(\boldsymbol{k}))}{\partial s(\boldsymbol{k})}\right\},
\end{aligned}
\end{aligned}
$$

where $\eta(\boldsymbol{k})$ and $\gamma(\boldsymbol{k})$ are the coefficients (17b) and (17c) in the kinetic equation (17). The $\operatorname{PDF} \mathcal{P}(s)$ at $k=3$ for four values of $\mathrm{H}_{2}$ is shown in Fig. 12. The PDF is obtained by the numerical integration of Eq. (27) until $100 T_{p}$. Figure 12 has $s$ normalized by its initial value $n(0)$, i.e., $x=s / n(0)$ on the abscissa. The variation of $\mathcal{P}(x)$ is faster for larger $H_{2}$. For $H_{2}=$ $1.25 \times 10^{-6}, \mathcal{P}(x)$ has a narrow distribution with a remnant of the initial distribution $\delta(x-1)$ even at $=100 T_{p}$, while for $H_{2}=1 \times 10^{-5}$, it has almost reached the $\chi^{2}$ distribution with 2 degrees of freedom corresponding to the Gaussianity of $a(\boldsymbol{k})$ by the same time. The PDF obtained in DNS at $100 T_{p}$ is also shown in Fig. 12. For all $\mathrm{H}_{2}$, the theoretical predictions of the RPA and the results of the DNS agree quite well. The RPA does not reply on any proximity to the Gaussianity of $a_{k}$ [12]. Figures 10, 11, and 12 confirm the validity of the RPA even when the Gaussianity of $a_{k}$ has not been established at all.

The coefficients $\eta(\boldsymbol{k})$ and $\gamma(\boldsymbol{k})$ in Eq. (27) vary as the wave action $n(\boldsymbol{k})$ evolves. Then, in the calculation of Eq. (27), the calculation of the kinetic equation (17a) is first performed to obtain $\gamma(\boldsymbol{k}, t)$ and $\eta(\boldsymbol{k}, t)$ as functions of time. Moreover, since $s(\boldsymbol{k})$ does not have fluctuations initially in this study, $\mathcal{P}(x, 0)=$ $\delta(x-1)$ should be employed for the initial condition to numerically obtain $\mathcal{P}(s)$ at a later time according to Eq. (27). However, it causes numerical difficulty. If $\mathcal{P}(x)$ is very narrow, the diffusion term is dominant in the right-hand side of Eq. (27). Then, in the very early stage of the evolution, Eq. (27) can be approximated by the diffusion equation

$$
\frac{\partial \mathcal{P}(x)}{\partial t}=\mu \frac{\partial^{2} \mathcal{P}(x)}{\partial x^{2}}, \quad \mu=\frac{x_{0} \eta_{0}}{n_{0}}, \quad x_{0}=1,
$$

where $\eta_{0}$ is $\eta$ at $t=0$. This makes it possible that the original initial condition $\mathcal{P}(x, 0)=\delta(x-1)$ is replaced by the state after the PDF evolves according to Eq. (28) for small time $t_{0}$, i.e., the normal distribution with small standard deviation $\sigma_{0}$,

$$
\mathcal{P}\left(x, t_{0}\right)=\frac{1}{\sqrt{2 \pi} \sigma_{0}} \exp \left(-\frac{(x-1)^{2}}{2 \sigma_{0}^{2}}\right) \quad\left(t_{0}=\sigma_{0}^{2} / 2 \mu\right),
$$

to numerically solve the initial-value problem of Eq. (27). For the result in Fig. 12, $\sigma_{0}=0.1$ is used. Even if $\sigma_{0}=0.05$ is used, a perceptible change in Fig. 12 is not produced. To obtain $\mathcal{P}(x)$ at $k=3$ in DNS, $s_{k}$ of 1680 modes in the annular domain $|k-3|<0.025$ are used. Since 256 realizations in DNS are independently performed, $430080(=1680 \times 256)$ data are used to draw $\mathcal{P}(x)$ in Fig. 12.

\section{CONCLUDING REMARKS}

In this work, DNS for a three-wave resonant Hamiltonian system are performed, and the validity of the RPA recently proposed in the weak turbulence theory is evaluated by quantitative comparison with DNS. It is confirmed that the theoretical predictions of the RPA and the results of the DNS agree quite well in all the statistical aspects of the amplitude fluctuations such as the high-order moments and the evolution of the PDF of $s(\boldsymbol{k})=|a(\boldsymbol{k})|^{2} / \delta(\boldsymbol{0})$ of each mode, the asymptotic approach of $a(\boldsymbol{k})$ to Gaussianity and its time scales, and so on. We have performed the comparison between the RPA and the DNS for the same three-wave model, but in the case of an anisotropic initial spectrum, and obtained the same level of agreement between the RPA and the DNS (not shown here).

The comparison is restricted to the three-wave resonant system in this work. In the RPA for four-wave systems, there exist some characteristics which the three-wave systems do not have such as the renormalization of the nonlinear frequency. Hence, the comparison for four-wave systems is still necessary. The authors numerically investigated the growth of the amplitude fluctuations in a four-wave system [19]. It was found that the time scales of the growth of the fluctuations are much shorter than those of the spectral variations in the large wave numbers away from the spectral peak. Although we did not attempt quantitative comparison with the RPA at that time, we felt suspicion about the validity of the RPA because the RPA predicts that both time scales should be of the same order in terms of the amplitude expansion or in terms of the Hamiltonian $H$. This suspicion was actually one of the motivations behind the present work. Judging from the complete agreement between the RPA and the DNS regarding the three-wave system reported here, we expect that this suspicion which we previously felt about the validity of the RPA would be cleared up when we have finished the same kind of comparison between the RPA and the DNS for some four-wave systems as well. In Ref. [19], we also investigated the statistical nature of $d s(\boldsymbol{k}) / d t$ by DNS. We numerically showed that the fluctuation of $d s(\boldsymbol{k}) / d t$ approaches a quasisteady state faster than $s(\boldsymbol{k})$ and analytically showed that the PDF of $d s(\boldsymbol{k}) / d t$ has the Laplace distribution. No work for $d s(\boldsymbol{k}) / d t$ was found in the framework of the RPA. This is also part of our future work.

The RPA gives theoretical predictions for the multimode statistics, such as the joint probability density $P^{(N)}$ of $\left|a_{\boldsymbol{k}_{1}}\right|^{2}, \ldots,\left|a_{\boldsymbol{k}_{N}}\right|^{2}$, as well as for the single-mode statistics [20], 
but we have restricted our attention in the present study to the single-mode statistics only and have not treated the multimode statistics at all. With regard to the multimode statistics, it is pointed out that the original results of the RPA are not correct [15]. Then, numerical investigation of the multimode statistics remains to be done.

Our present numerical results also prompt a new question of the time scales which is required by the weak turbulence theory. Janssen [21] derived a kinetic equation in a form different than that of Hasselmann [1] and that of Zakharov et al. [11]. He pointed out that the conventional kinetic equation is recovered by replacing the "resonance function"

$$
R_{i}(\Delta \omega, t)=\frac{\sin (\Delta \omega t)}{\Delta \omega}
$$

by the $\delta$ function which is the asymptotic of $R_{i}$ in the limit $t \rightarrow \infty$. He claimed that nonresonant interactions as well as resonant interactions contribute the spectral evolution until the time when the replacement is validated. In the RPA investigated here, the important equations, such as for the moments, Eq. (20), and for the PDF, Eq. (27), are derived. It is based on the premise that the linear time scale, $\tau_{l}=O(2 \pi / \omega)$, and the nonlinear time scale, i.e., the time scale of the spectral evolution, $\tau_{n}=O\left(1 /\left(\epsilon^{2} \omega\right)\right)$, are well separated, and the intermediate time scale,

$$
\tau_{l} \ll \tau_{i} \ll \tau_{n}
$$

should exist. Based on their derivation, the equations in the RPA are supposed to describe the time rate of change of the moments and the PDF in the time scale of $\tau_{i}$.

In Fig. 13, the time rates of spectral changes $d E(k) / d t$ during $T_{p}$ from $t=0$ to $t=T_{p}$ and from $t=T_{p}$ to $t=2 T_{p}$ for $H_{2}=5 \times 10^{-6}$ are compared with those obtained by the kinetic equation (17) for the initial spectrum. Although the time rate of the spectral change for the first $T_{p}$ is slightly different from the prediction of the kinetic equation, the rate

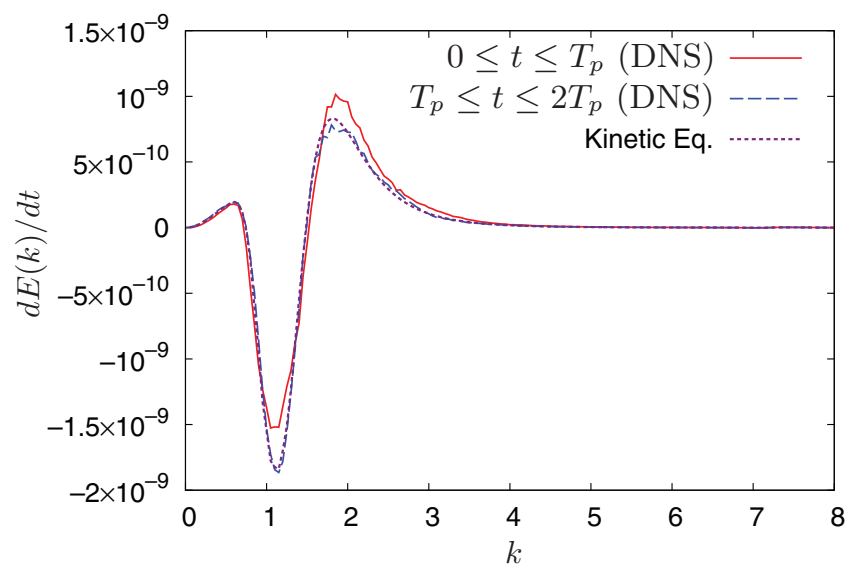

FIG. 13. (Color online) Comparison of $d E(k) / d t$ obtained from the spectral variation during one period in the DNS and from the kinetic equation.

during the period $T_{p}$ from $t=T_{p}$ to $t=2 T_{p}$ almost perfectly coincides with the prediction of the kinetic equation. The time rates of change of the higher-order moments during $T_{p}$ also show similar agreements between the DNS and the RPA. These agreements between the DNS and the RPA in the short-time evolution appear to contradict the procedure of the derivation of the statistical equations in the RPA. This short-time agreement has already been pointed out previously [22]. This might affect the basis of the weak turbulence theory, including the RPA, and must be investigated further.

\section{ACKNOWLEDGMENTS}

The authors are grateful to Dr. Yuri V. Lvov and Dr. Yeontaek Choi for valuable discussions. The numerical simulations were performed at the Information Technology Center, Nagoya University.
[1] K. Hasselmann, J. Fluid Mech. 12, 481 (1962).

[2] V. E. Zakharov and N. N. Filonenko, Sov. Phys. Dokl. 10, 881 (1967).

[3] Y. Lvov, S. Nazarenko, and R. West, Physica D 184, 333 (2003).

[4] C. Connaughton, S. Nazarenko, and A. C. Newell, Physica D 184, 86 (2003).

[5] E. Kartashova, Nonlinear Resonance Analysis: Theory, Computation, Applications (Cambridge University Press, Cambridge, UK, 2010).

[6] A. C. Newell, S. Nazarenko, and L. Biven, Physica D 152-153, 520 (2001).

[7] S. Dyachenko, A. C. Newell, A. Pushkarev, and V. E. Zakharov, Physica D 57, 96 (1992).

[8] A. C. Newell and B. Rumpf, Annu. Rev. Fluid Mech. 43, 59 (2011).

[9] A. N. Pushkarev, Eur. J. Mech., B/Fluids 18, 345 (1999).

[10] G. Düring, C. Josserand, and S. Rica, Phys. Rev. Lett. 97, 025503 (2006).
[11] V. E. Zakharov, V. S. L'vov, and G. Falkovich, Kolmogorov Spectra of Turbulence I (Springer-Verlag, Berlin, 1992).

[12] Y. V. Lvov and S. Nazarenko, Phys. Rev. E 69, 066608 (2004).

[13] S. Nazarenko, Wave Turbulence (Springer, Heidelberg, 2011).

[14] Y. Choi, Y. V. Lvov, S. Nazarenko, and B. Pokorni, Phys. Lett. A 339, 361 (2005).

[15] G. L. Eyink and Y.-K. Shi, Physica D 241, 1487 (2012).

[16] D. J. Benney and A. C. Newell, Stud. Appl. Math. 48, 29 (1969).

[17] D. J. Benney and P. G. Saffman, Proc. R. Soc., Ser. A 289, 301 (1966).

[18] C. Canuto, M. Y. Hussaini, A. Quarteroni, and T. A. Zang, Spectral Methods in Fluid Dynamics (Springer-Verlag, Berlin, 1988).

[19] M. Tanaka and N. Yokoyama, Physica D 240, 1145 (2011).

[20] Y. Choi, Y. V. Lvov, and S. Nazarenko, Physica D 201, 121 (2005).

[21] P. A. E. M. Janssen, J. Phys. Oceanogr. 33, 863 (2003).

[22] M. Tanaka, J. Phys. Oceanogr. 37, 1022 (2007). 